FERREIRA, P.G. et al. Soroepidemiologia da brucelose bovina no período de 2010 a 2012 no Município de Alegre/Espírito Santo. PUBVET, Londrina, V. 8, N. 11, Ed. 260, Art. 1726, Junho, 2014.

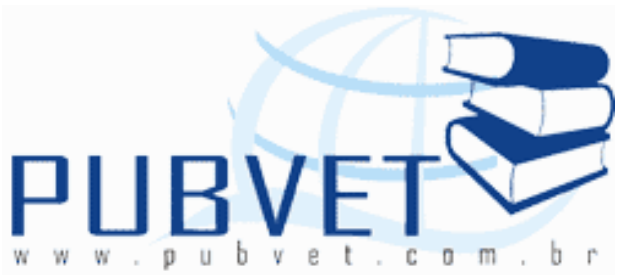

PUBVET, Publicações em Medicina Veterinária e Zootecnia.

\title{
Soroepidemiologia da brucelose bovina no período de 2010 a 2012 no Município de Alegre/Espírito Santo
}

\begin{abstract}
Peter Gabriel Ferreira1, Igor Luiz Salardani Senhorello1, Alexandre de Oliveira Bezerra1, Renan Zappavigna Costa Starling1, Dirlei Molinari Donatele1, Renata Côgo Clipes²
\end{abstract}

1 Universidade Federal do Espírito Santo, Departamento de Medicina Veterinária; Alegre; Espírito Santo; Brasil.

${ }^{2}$ Instituto Federal do Espírito Santo; IFES-Campus Alegre; Alegre; Espírito Santo; Brasil.

\section{Resumo}

Objetivou-se avaliar a soroprevalência da brucelose bovina e os fatores de risco associados no rebanho bovino do município de Alegre, Espírito Santo, no período de 2010 a 2012. Foram analisadas 516 amostras sanguíneas de bovinos com idade maior que 24 meses, de 26 propriedades rurais. 0 diagnóstico foi feito por meio do método indireto de diagnóstico e triagem, previsto oficialmente pelo Programa Nacional de Controle e Erradicação da Brucelose e Tuberculose (PNCEBT), Antígeno Acidificado Tamponado (AAT). Das 516 amostras testadas, 07 foram soros-reagentes, totalizando uma soroprevalência de $1,35 \%$, presentes em $23,1 \%$ das propriedades. Das variáveis: assistência médico veterinária; compra e venda de animais; vacinação contra brucelose; conhecimento do PNCEBT; e testagem dos animais 
FERREIRA, P.G. et al. Soroepidemiologia da brucelose bovina no período de 2010 a 2012 no Município de Alegre/Espírito Santo. PUBVET, Londrina, V. 8, N. 11, Ed. 260, Art. 1726, Junho, 2014.

para brucelose, não apresentaram diferença significativa $(p>0,05)$ para ocorrência da brucelose. Em 42,3\% (11) das propriedades não era realizada a vacinação contra brucelose, $84,6 \%$ (22) realizavam compra e venda de animais, $57,7 \%$ (15) não testavam os animais para brucelose, 53,9\% (14) dos responsáveis pela propriedade não tinham conhecimento do PNCEBT e apenas 38,5\% (11) possuíam assistência do médico veterinário. Esses resultados demonstram que a infecção pela Brucella abortus está distribuída no rebanho leiteiro do município de Alegre, Espírito Santo. Atentando-se ao carácter ocupacional da doença, uma vez que pessoas que lidam com animais susceptíveis à doença e/ou infectados, o risco de se infectarem é maior, o contato direto com anexos fetais, restos fetais e fluidos propicia que esta zoonose se dissemine. Torna-se imprescindível que medidas sejam adotadas para que os proprietários rurais recebam esclarecimentos sobre a doença e suas implicações econômicas, visando à implantação de um programa de controle da doença na região.

Palavras-chave: Brucelose, bovinos, epidemiologia

\section{Serum epidemiology of bovine brucellosis in the period from 2010 to 2012 in the municipality of Alegre / Espírito Santo}

\section{Abstract}

The purpose of this work was to evaluate the seroprevalence of brucellosis and associated risk factors in cattle in the municipality of Alegre, Espírito Santo, in the period 2010-2012. We analyzed 516 blood samples from cattle older than 24 months of 26 farms. The diagnosis was made by means of the indirect method of diagnosis and screening provided officially by the National Program for the Control and Eradication of Brucellosis and Tuberculosis ( PNCEBT), Buffered Acidified Antigen ( AAT ). Of the 516 samples tested, 07 sera were reactive, with a total prevalence of $1.35 \%$, present in $23.1 \%$ of the properties. Variables: veterinary medical care, buying and selling animals, vaccination against brucellosis; knowledge PNCEBT, and testing of animals for 
FERREIRA, P.G. et al. Soroepidemiologia da brucelose bovina no período de 2010 a 2012 no Município de Alegre/Espírito Santo. PUBVET, Londrina, V. 8, N. 11, Ed. 260, Art. 1726, Junho, 2014.

brucellosis, showed no significant difference $(p>0.05)$ for the occurrence of brucellosis. In $42.3 \%$ ( 11 ) of the properties was not made vaccination against brucellosis, $84.6 \%$ ( 22 ) did buying and selling animals, $57.7 \%$ ( 15 ) not tested the animals for brucellosis, $53.9 \%$ ( 14 ) responsible for the property were not aware of PNCEBT and only $38.5 \%$ ( 11 ) had assistance from a veterinarian. These results demonstrate that infection by Brucella abortus is distributed in the dairy herd of the municipality of Alegre, Espírito Santo. Paying attention to the occupational nature of the disease, since people who deal with animals susceptible to the disease and / or infected, the risk of becoming infected is greater, direct contact with fetal membranes, fetal remains fluid and provides that this will spread zoonosis. It is essential that measures be adopted to ensure that landowners receive information on the disease and its economic implications, aiming at the implementation of a program to control the disease in the region.

Keywords: Bovine, brucellosis, epidemiology

\section{Introdução}

A brucelose bovina é uma importante enfermidade infectocontagiosa, que, a despeito dos esforços voltados para o seu controle, ainda se constitui um problema econômico e sanitário dos mais sérios para os rebanhos bovinos de diversos países, entre os quais o Brasil (MATHIAS et al., 2001).

Os programas que têm obtido sucesso no combate a essa enfermidade baseiam-se principalmente em provas sorológicas com eliminação dos animais reagentes e na vacinação de bezerras entre 3 e 8 meses de idade (MEGID et al., 2000).

Em levantamentos epidemiológicos no Brasil a incidência da doença está em torno de $5 \%$ do rebanho efetivo (BRASIL, 2006), e isto acarreta um grande impacto na economia do país, uma vez que acomete o rebanho de um dos principais exportadores de carne bovina do mundo. 
FERREIRA, P.G. et al. Soroepidemiologia da brucelose bovina no período de 2010 a 2012 no Município de Alegre/Espírito Santo. PUBVET, Londrina, V. 8, N. 11, Ed. 260, Art. 1726, Junho, 2014.

Segundo AZEVEDO et al. (2009) o Estado do Espírito Santo mantém sua prevalência em torno de 9,0\% das propriedades analisadas, sendo que de 1975 até 2009 manteve em 9,6\%, e a região sul do Estado do Espírito Santo, na qual se encontra o município de Alegre, a prevalência nas propriedades analisadas foi de $10,86 \%$.

Estudos feitos em países com sistema de controle consistentes revelam que a brucelose é responsável pela diminuição da produção de leite e carne em $25 \%$, redução de $15 \%$ na produção de bezerros e aumento dos intervalos entre partos (MONTEIRO, 2004), revelando que em cada cinco vacas soropositivas uma aborta ou se torna permanentemente estéril (BRASIL, 2006).

Em um rebanho bovino com 212,8 milhões de cabeças, os prejuízos econômicos estão estimados em mais 140 milhões de reais aos produtores do Brasil, segundo Santos et al. (2013), e o Estado do Espírito Santo com um rebanho de 2.223 .262 bovinos (IBGE, 2011) os prejuízos podem chegar a mais $6.246 .341,29$ reais (SANTOS et al., 2013).

O presente trabalho teve como objetivo fazer um levantamento epidemiológico da brucelose nas propriedades de gado bovino do município de Alegre/ES, no período de 2010 a 2012, e associar os principais fatores de risco da doença na região.

\section{Materiais e Métodos}

Foram visitadas 26 propriedades distribuídas no município de Alegre, ao Sul do Estado do Espírito Santo. O presente município possui 52.247 cabeças de bovinos, sendo o quinto maior rebanho bovino do Estado, possui uma área territorial de $772,7 \mathrm{~km}^{2}$ (IBGE, 2010) e sua economia é baseada em atividades agropecuárias, onde predomina a pecuária leiteira e a cafeicultura.

Para o agendamento das visitas, utilizou-se listagem obtida por meio do cadastro do Instituto de Defesa Agropecuária e Florestal do Espírito Santo (IDAF-ES) e da Secretaria Municipal de Agricultura, sorteada de forma 
FERREIRA, P.G. et al. Soroepidemiologia da brucelose bovina no período de 2010 a 2012 no Município de Alegre/Espírito Santo. PUBVET, Londrina, V. 8, N. 11, Ed. 260, Art. 1726, Junho, 2014.

aleatória. O Termo de Consentimento e Livre Esclarecido do projeto foi apresentado e assinado pelo responsável pela propriedade.

Foram avaliados 516 bovinos de corte e de leite acima de 24 meses, pertencentes a 26 propriedades distintas, no período de 2010 a 2012. Coletouse, no mínimo, uma amostra de $10 \%$ do total de bovinos das propriedades.

Previamente à coleta das amostras foi aplicada uma entrevista ao proprietário ou responsável pela propriedade, com o objetivo de identificar, e quantificar os produtores e propriedades visitadas e os animais do estudo. Na entrevista, foram apurados dados a respeito do manejo sanitário do rebanho, (assistência médico veterinária, compra e venda de animais, conhecimento do PNCEBT, prática da vacinação e testagem dos animais).

As amostras de sangue foram coletadas, por meio de venopunção da veia coccígea, utilizando-se um sistema a vácuo (vacutainer $®$ ), identificados, armazenados em tubos de $10 \mathrm{~mL}$ sem anticoagulantes, e transportada em caixa isotérmica com gelo ao Laboratório de Inspeção de Produtos de Origem Animal (LIPOA) do Hospital Veterinário da Universidade Federal do Espírito Santo.

As amostras coletadas foram centrifugadas por 10 minutos a 1600 rotações por minuto, para a obtenção do soro. Posteriormente, os soros foram transferidos para tubos tipo eppendorf $\AA$, os quais foram identificados e mantidos a temperatura de $-20^{\circ} \mathrm{C}$, até o processamento do mesmo.

Para o diagnóstico de brucelose foi utilizado o teste do Antígeno Acidificado Tamponado (AAT), recomendada pelo Manual Técnico do Programa Nacional de Controle e Erradicação da Brucelose e Tuberculose (BRASIL, 2006).

Os dados foram tabulados em planilhas do Microsoft Excel 2007. A prevalência foi obtida pela proporção de animais soros-reagentes entre a totalidade dos animais testados.

A analise estatística foi realizada pelo programa Open Epi, Versão 2. Foi utilizada a estatística descritiva e as associações entre as variáveis (soro e 
FERREIRA, P.G. et al. Soroepidemiologia da brucelose bovina no período de 2010 a 2012 no Município de Alegre/Espírito Santo. PUBVET, Londrina, V. 8, N. 11, Ed. 260, Art. 1726, Junho, 2014.

variáveis) foram testadas pelo Qui-quadrado $\left(X^{2}\right)$, com nível de significância de $5 \%$.

\section{Resultados e discussão}

Dos 516 animais testados de 26 propriedades do município de Alegre, Espírito Santo, 1,35\% (7) foram soros-reagentes para brucelose bovina, distribuídas em $23,1 \%$ (6) das propriedades estudadas. Vinte propriedades foram consideradas negativas para brucelose, pois não apresentavam animal soropositivo, conforme descrito na Tabela 1.

Tabela 1. Amostras de soro de bovinos adquiridas de 26 propriedades distintas e testadas com Antígeno Acidificado Tamponado (AAT) para brucelose (Brucella abortus), no município de Alegre, ES, 2010-2012.

\begin{tabular}{|c|c|c|c|}
\hline \multirow{3}{*}{ Propriedade } & \multicolumn{3}{|c|}{ Amostras Examinadas } \\
\hline & \multirow{2}{*}{ № de Amostras } & \multicolumn{2}{|c|}{ Soro-reagente } \\
\hline & & № & $(\%)$ \\
\hline$A$ & 122 & 1 & 0,82 \\
\hline $\mathrm{B}$ & 4 & - & - \\
\hline C & 11 & 1 & 9,1 \\
\hline $\mathrm{D}$ & 89 & 1 & 1,12 \\
\hline$E$ & 37 & - & - \\
\hline $\mathrm{F}$ & 1 & - & - \\
\hline G & 14 & - & - \\
\hline $\mathrm{H}$ & 4 & - & - \\
\hline 1 & 7 & - & - \\
\hline $\mathrm{J}$ & 29 & - & - \\
\hline $\mathrm{K}$ & 30 & 1 & 3,33 \\
\hline $\mathrm{L}$ & 10 & - & - \\
\hline $\bar{M}$ & 12 & - & - \\
\hline $\mathrm{N}$ & 13 & - & - \\
\hline $\mathrm{O}$ & 9 & - & - \\
\hline$P$ & 25 & 2 & 8 \\
\hline$Q$ & 6 & - & - \\
\hline $\mathrm{R}$ & 10 & - & - \\
\hline$S$ & 23 & - & - \\
\hline $\mathrm{T}$ & 19 & 1 & 5,26 \\
\hline$U$ & 25 & - & - \\
\hline $\mathrm{V}$ & 2 & - & - \\
\hline W & 3 & - & - \\
\hline$x$ & 4 & - & - \\
\hline$Y$ & 4 & - & - \\
\hline Z & 3 & - & - \\
\hline TOTAL & 516 & 7 & 1,35 \\
\hline
\end{tabular}


FERREIRA, P.G. et al. Soroepidemiologia da brucelose bovina no período de 2010 a 2012 no Município de Alegre/Espírito Santo. PUBVET, Londrina, V. 8, N. 11, Ed. 260, Art. 1726, Junho, 2014.

As principais informações adquiridas, por meio das entrevistas realizadas em cada propriedade visitada estão descritas na Tabela 2 e Tabela 3.

Tabela 2. Distribuição de frequência de bovinos soro-reagente ao AAT (Antígeno Acidificado Tamponado) em relação às variáveis estudadas no município de Alegre/ES, no período de 2010 a 2012.

\begin{tabular}{|c|c|c|c|c|c|c|c|}
\hline \multirow{2}{*}{ Variáveis } & & \multicolumn{4}{|c|}{ Bovinos } & \multirow[b]{2}{*}{$P$} & \multirow[b]{2}{*}{ OR } \\
\hline & & Positivos & $(\%)$ & Negativos & $(\%)$ & & \\
\hline \multirow{2}{*}{$\begin{array}{l}\text { Assistência } \\
\text { Veterinária }\end{array}$} & Sim & 05 & 0,97 & 339 & 65,70 & \multirow{2}{*}{0,8930} & \multirow{2}{*}{1,254} \\
\hline & Não & 02 & 0,39 & 170 & 32,94 & & \\
\hline \multirow{2}{*}{$\begin{array}{l}\text { Compra e } \\
\text { Venda de } \\
\text { Animais }\end{array}$} & Sim & 05 & 0,97 & 366 & 70,93 & \multirow{2}{*}{0,8930} & \multirow{2}{*}{0,9768} \\
\hline & Não & 02 & 0,39 & 143 & 27,71 & & \\
\hline \multirow{2}{*}{$\begin{array}{l}\text { Vacinação dos } \\
\text { animais }\end{array}$} & Sim & 02 & 0,39 & 94 & 18,21 & \multirow{2}{*}{0,5168} & \multirow{2}{*}{1,766} \\
\hline & Não & 05 & 0,97 & 415 & 80,43 & & \\
\hline \multirow{2}{*}{$\begin{array}{l}\text { Animais } \\
\text { testados }\end{array}$} & Sim & 01 & 0,19 & 257 & 49,81 & \multirow{2}{*}{0,8467} & \multirow{2}{*}{0,1634} \\
\hline & Não & 06 & 1,17 & 252 & 48,83 & & \\
\hline
\end{tabular}

$\mathrm{P}=$ Probabilidade de ocorrência ao acaso; OR = Odds ratio (95\%);

Tabela 3. Frequência de propriedade de bovinos avaliada quanto a presença da brucelose em relação às variáveis estudadas no município de Alegre, ES, 2011.

\begin{tabular}{|c|c|c|c|c|}
\hline \multirow[b]{2}{*}{ Variáveis } & & \multicolumn{3}{|c|}{ Propriedades } \\
\hline & & $\begin{array}{c}\text { Negativas para } \\
\text { Brucelose } \\
\text { (n / \%) }\end{array}$ & $\begin{array}{l}\text { Positivas* } \\
\text { para } \\
\text { Brucelose } \\
\text { (n/\%) }\end{array}$ & $\begin{array}{l}\text { Total } \\
\text { (n / \%) }\end{array}$ \\
\hline \multirow{2}{*}{ Assistência Veterinária } & Sim & $06 / 23,1$ & $04 / 15,4$ & $11 / 38,5$ \\
\hline & Não & $14 / 53,8$ & $02 / 7,7$ & $12 / 61,5$ \\
\hline \multirow{2}{*}{ Compra e Venda de Animais } & Sim & $16 / 61,5$ & $06 / 23,1$ & $22 / 84,6$ \\
\hline & Não & $04 / 15,4$ & - & $04 / 15,4$ \\
\hline \multirow{2}{*}{ Conhecimento do PNCEBT } & Sim & $09 / 34,7$ & $03 / 11,5$ & $12 / 46,1$ \\
\hline & Não & $11 / 42,3$ & $03 / 11,5$ & $14 / 53,9$ \\
\hline \multirow{2}{*}{ Vacinação dos animais } & Sim & $12 / 46,2$ & $03 / 11,5$ & $15 / 57,7$ \\
\hline & Não & $08 / 30,8$ & $03 / 11,5$ & $11 / 42,3$ \\
\hline \multirow{2}{*}{ Animais testados } & Sim & $08 / 30,8$ & $03 / 11,5$ & $11 / 42,3$ \\
\hline & Não & $12 / 46,2$ & $03 / 11,5$ & $15 / 57,7$ \\
\hline
\end{tabular}

*Propriedades Positivas para Brucelose = presença de pelo menos um animal soro-reagente ao teste de AAT. 
FERREIRA, P.G. et al. Soroepidemiologia da brucelose bovina no período de 2010 a 2012 no Município de Alegre/Espírito Santo. PUBVET, Londrina, V. 8, N. 11, Ed. 260, Art. 1726, Junho, 2014.

A soroprevalência de $1,3 \%$ encontrada no município de Alegre/ES é inferior a média nacional que é de 7,5\% (BRASIL, 2006), e aos 3,5\% do Espírito Santo, descrito por Azevedo et al. (2009), sendo que os mesmos autores relataram uma prevalência de $3,69 \%$, a qual é bem superior a encontrada no município de Alegre/ES.

Mota (2008) realizou um levantamento da prevalência da brucelose bovina no rebanho do Espírito Santo e identificou um total de 81 propriedades com ao menos um animal positivo, das 1.067 propriedades analisadas. Verificou um montante de 299 bovinos soro-reagentes ao teste do AAT, dos 21.700 testados, obtendo uma prevalência de 1,4\% em 2007 no Estado do Espírito Santo.

O valor de prevalência encontrado no município de Alegre, ES se assemelha com 0 de estados brasileiros com controle para brucelose considerado ótimo, como Minas Gerais (1,09\%), Rio Grande do Sul (1,01\%) (BRASIL, 2006), Distrito Federal (0,14\%) (GONÇALVES et al., 2009) e Santa Catarina $(0,06 \%)$ (SIKUSAWA et al., 2009).

As variáveis avaliadas: assistência médico veterinária; compra e venda de animais; vacinação contra brucelose; conhecimento do PNCEBT; e testagem dos animais para brucelose, não apresentaram diferença significativa ( $p>$ 0,05) para ocorrência da brucelose.

A variável presença do médico veterinário esteve em 38,5\% das propriedades (Tabela 3), e 40\% destas apresentaram ao menos 01 (um) animal positivo para brucelose. Esta variável se assemelhou aos valores encontrados por Azevedo et al. (2009), quando 36,2\% das propriedades do estrato sul do Estado eram assessoradas. A presença dos profissionais da área é fundamental, visto que, podem contribuir para agilizar e notificar a ocorrência de sinais clínicos da infecção por Brucella abortus com posterior investigação dos casos.

A compra e venda de animais é realizada no município de Alegre/ES de forma indiscriminada, sem controle sanitário adequado para tal atividade. Esta prática esteve presente em $84,6 \%$ das propriedades estudadas, e não foi 
FERREIRA, P.G. et al. Soroepidemiologia da brucelose bovina no período de 2010 a 2012 no Município de Alegre/Espírito Santo. PUBVET, Londrina, V. 8, N. 11, Ed. 260, Art. 1726, Junho, 2014.

considerado fator de risco para a ocorrência da brucelose, porém em muitos trabalhos é considerado fator promotor de disseminação da doença. A introdução de animal(is) exógeno(s) numa propriedade livre é de fato arriscado, outrora este(s) animais podem estar infectados, todavia o risco pode variar de acordo com a fonte (CRAWFOR; HUBER; ADAMS, apud AZEVEDO, 2009).

Kellar, Marra e Martin (1976), em Ontario, Canadá observaram que rebanhos os quais proprietários compravam bovinos com frequência apresentaram um risco elevado de serem foco da brucelose. Azevedo (2006) revelou, em seu estudo, esta prática como fator de risco, com um valor de OR muito elevado $(16,7$ : IC $95 \%=1,51-184,58)$. Este fato reforça a necessidade de intensificar estratégias de ação do PNCEBT, principalmente nos casos de trânsito de animais destinados à reprodução e nos casos de exposições, onde ocorre a aglomeração destes.

Nesta análise foi notado que 53,9\% dos proprietários não tinham conhecimento do PNCEBT. Foi uma variável que não se associou à presença ou não da doença nas propriedades, porem notou-se que esta ferramenta não está sendo praticada pelos responsáveis das propriedades.

Vacinação ou não dos animais no estudo não se comportou como fator de risco para ocorrência da brucelose, porém, os dados podem não ser tão confiáveis, uma vez que dependem da memória do informante e os proprietários se sentem sem credibilidade caso confirmem a não vacinação de seus animais. Provavelmente, a vacinação não leva à erradicação da brucelose por si só, mas é o primeiro passo no início do saneamento de uma propriedade. Todavia a vacinação estratégica é um grande fator positivo para a não ocorrência da enfermidade.

Lage et al. (2005) relatam que a vacinação de fêmeas entre três e oito meses com a vacina B19, associada a vacinação estratégica de fêmeas com idade aleatória superior a 8 meses, não vacinas com a B19, com a RB51 acarreta uma boa cobertura vacinal, e proporciona índices menores de 
FERREIRA, P.G. et al. Soroepidemiologia da brucelose bovina no período de 2010 a 2012 no Município de Alegre/Espírito Santo. PUBVET, Londrina, V. 8, N. 11, Ed. 260, Art. 1726, Junho, 2014.

percentagem de animais susceptíveis, de aborto e consequentemente a taxa de infecção também diminui.

A cobertura vacinal do presente trabalho se apresentou muito baixa $(18,8 \%)$, este valor implica em 97 animais, quando analisada proporcionalmente, o número de animais vacinados na análise foge do ideal para um excelente controle e combate à brucelose de acordo com Azevedo et al. (2009).

A variável; animais testados ou não para a brucelose também não indicou valores que induzem a presença ou não da bactéria no rebanho neste estudo. Em levantamento feito por Azevedo (2006) no Espírito Santo dividiu-se o estado em dois estratos de estudo (estrato 1- Norte e estrato 2- Sul), onde no estrato sul, $21,5 \%$ das propriedades realizavam testes diagnósticos para a doença, e no presente estudo, $42,3 \%$ das propriedades realizam teste diagnóstico.

A brucelose está presente no rebanho bovino do município de Alegre, ES e é fator de relevância, uma vez que se trata de uma enfermidade que acarreta prejuízos econômicos e riscos á saúde animal e humana.

O diagnóstico sorológico para brucelose é de extrema importância para estratégias e certificação de propriedades livres e monitoradas, na qual todos os animais soropositivos são eutanasiados. Com esta prática, esforços devem ser concentrados para conscientizar proprietários sobre a importância da aplicação de testes diagnósticos nas propriedades para que se atinja o controle da doença.

Nas propriedades classificadas como positivas, cabe ao profissional sanitarista informar e orientar no sentido de evitar a manutenção da brucelose nos rebanhos que possuem animais infectados, pois se sabe que essa se deve, principalmente, às falhas de manejo, dos partos distócicos, destino de restos de abortos e partos dos animais doentes (LUNA-MARTÍNEZ; MEJÍA-TERÁN, 2002). 


\section{Referências Bibliográficas}

ACHA,P.N.; SZYFRES,B. Zoonosis y enfermidades transmisibles comunes al hombre y animales. 2.ed. Washington: Organization Panamericana de la Salud, 1986.

ACHA, P.N.; SZYFRES, B. Zoonoses e enfermidades transmissíveis comuns ao homem e aos animais: Bacterioses e micoses. 3.ed. Washington: OPAS, p. 416. 2001

ALTON, G.G.; JONES, L.M.; ANGUS, R.D.; VERGER, J.M. Techniques for the brucellosis laboratory. Paris: INRA, p. 188.1988.

ASHFORD, D.A.; DI PIETRA, J.; LINGAPPA, J. Adverse events in humans associated with accidental exposure to the livestock brucellosis vaccine RB51,Vaccine, v. 22, n. 25-26, p.3435-3439, 2004.

AZEVEDO, S.S.; NETO, J.S.F.; DIAS, R.A.; FERREIRA, F.; AMAKU, M.; FIGUEIREDO, V.C.F.; LÔBO, J.R.; GONÇALVES, V.S.P.; SOUZA, A.C.; VASCONCELLOS S.A. Situação epidemiológica da brucelose bovina no Estado do Espírito Santo. Arquivo Brasileiro Medicina Veterinária Zootecnia, v.61, supl. 1, p.19-26, 2009.

BEER, J. (Ed.). Doenças infecciosas em animais domésticas. São Paulo: Editora Roca, 1988 , p. 380.

BEER, J. Doenças Infecciosas em animais domésticos. São Paulo: Roca, 1999. p 163-178.

BEVILACQUA, M.R. Brucelose em bovinos. 2008, 28 f. Monografia (Pós-graduação em Medicina Veterinária) - Universidade Castelo Branco, Campo Grande, Mato Grosso do Sul.

BLAHA, T. Epidemiologia especial veterinária. Saragoza, Espanha: Ed. Acribia. 1995. 529 p.

Brasil. International Atomic Energy Agency, Vienna, IAEA-TECDOC-1055, p. 63-68.1998.

BRASIL. Ministério da Agricultura, Pecuária e Abastecimento. Manual Técnico do Programa Nacional de Controle e Erradicação da Brucelose e da Tuberculose Bovina (PNCEBT). 2006. Disponível em: <http://www.agricultura.gov.br/>. Acesso em: 21 jan. 2013.

CAMPAÑA, R.C.; GOTARDO, D.J. e ISHIZUKA, M.M. Epidemiologia e profilaxia da brucelose bovina e bubalina. Coordenadoria de Defesa Agropecuária de São Paulo. 2003. Disponível em: http://www.cda.sp.gov.br/DocEst/Docs/bru/info doc bru1.htm. Acessado em: 10 de fev. 2013.

CHOSEWOOD, L.C.; WILSON, D.E. Biosafety in microbiological and biomedical laboratories. Washington: US Government Printing Office. p. 409. 2007.

CORRÊA, W.M.; CORRÊA, C.N.M. Enfermidades dos mamíferos domésticos. 2. ed. Rio de Janeiro, Brasil: Médica e Científica, 1992. p. 195-823.

CRAWFORD, R.P., HUBER, J.D.; ADAMS, B.S. Epidemiology and surveillance. In: Nielsen K, Duncan JR (Ed.). Animal Brucellosis. Boca Raton: CRC Press, 1990. p.131-151. 
CRAWFORD, P.R.; HUBER, J.D.; ADAMS, B.S.; EAGLESOME, M.D.; GARCIA, M.M. Epidemiology and surveillance. In: Microbial agents associated with bovine genital tract infection and semen. Parte 1. Brucella abortus, Leptospira, Campylobacter fetus and Trichomonas foetus. Vet Bull, v. 62, p.743-775, 1992.

EAGLESOME, M.D.; GARCIA, M.M. Microbial agents associated with bovine genital tract infections and semen. Part I. Brucella abortus, Leptospira, Campylobacter fetus and Tritrichomonas foetus. Vet Bull, v.62, p.743-753, 1992.

FERREIRA, A. J.; FERREIRA, C. Doenças infecto-contagiosas dos animais domésticos. 4. ed. Fundação Calouste Gulbenkian, Lisboa: 1990. p. 125-143.

GERMANO, P.M.L.; GERMANO, M.I.S. Brucelose. In: de Alimentos. São Paulo: Livraria Varela, 2003. p. 277-282. Higiene e Vigilância Sanitária

GODFROID, J.; CLOECKAERT, A.; LIAUTARD, J.P.; KOHLER, S. From the discovery of the Malta fever's agent to the discovery of a marine mammal reservoir, brucellosis has continuously been a re-emerging zoonosis. Veterinary Research, v.36, p. 313-326, 2005.

GONÇALVES, V.S.P; RIBEIRO, L.A.; CALDAS, R.A.; FRANCISCO, P.F.C Situação epidemiológica da brucelose bovina no Distrito Federal. Arquivo Brasileiro de Medicina Veterinária e Zootecnia. v.61, supl.1, p.14-18, 2009.

GORVEL, J.P.; MORENO, E. Brucella intracelular: de invasão a replicação intracelular. Microbiologia Veterinária, v. 90, p.281-297, 2002.

HIRSH, D.C.; ZEE, Y.C. Microbiologia Veterinária. Rio de Janeiro: Guanabara Koogan, 2003. p.191-195.

INSTITUTO BRASILEIRO DE GEOGRAFIA E ESTATÍSTICA. Produção agropecuária municipal2011. Acessado em 19 de julho de 2013. Disponível em:

ftp://ftp.ibge.gov.br/Producao_Pecuaria/Producao_da_Pecuaria_Municipal/2011/tabelas_pdf/ta $\underline{\text { b01.pdf }}$

ITO, F.H.; VASCONCELLOS, S.A.; BERNARDO, F.; NASCIMENTO, A.A.; LABRUNA, M.B.; ARANTES, I.G. Evidência sorológica de brucelose e leptospirose e parasitismo por ixodídeos em animais silvestres do Pantanal Sul-mato-grossense. Ars Veterinária. v. 14, n. 3, p.302-310. 1998.

KELLAR, J.; MARRAR, R.; MARTIN, W. Brucellossis in Ontario: a cause control study. Canidian Journal of Comparative Medicine, v. 40, p. 119-128, 1976.

LAGE, A.P.; POESTE,R.F.P; GONÇALVES, V.S.P.; ROXO, E.; MÜLLER. E.E.; CAVALLÉRO, J.C.M.; FERREIRA-NETO, J.S.; MOTTA, P.M.P.C.; FIGUEIREDO, V.C.F.; LÔBO, J.R. Programa nacional de controle e erradicação da brucelose e tuberculose. Caderno Técnico de Veterinária e Zootecnia, n.47, p.99-110, 2005.

LAGE, A.P.; POESTER, F.P.; PAIXÃO T.A.; TEANE, M.A.S.; XAVIER, M.N.; SÍLVIA M.; MIRANDA, K.L.; ALVES, C.M.; MOL, J.P.S.; SANTOS, R.L. Brucelose bovina: uma atualização. Revista Brasileira de Reprodução Animal. Belo Horizonte, v.32, n.3, p.202-212, 2008.

LAMONTAGNE, A.; FOREST, E.; MARAZZO, F.; DENIS, H.; BUTLER, J.F.; MICHAUD, L.; BOUCHER, I.; PEDRO, A.; VILLENEUVE, D.; SITNIKOV, K.; TRUDEL, N.; NASSIF, D.; BOUDJELT, F.; TOMAKI, E.; CHAVES-OLARTE, C.; GUZMAN-VERRI, S.; BRUNET, A. ; COTE- 
MARTIN, J.; HUNTER, E.; MORENO, E.; PARAMITHIOTIS, E. Intracellular adaptation of Brucella abortus. Journal of Proteome Research, Washington, v. 8, n. 3, p. 1594-1609, 2009.

LUNA-MARTÍNEZ, J.E.; MEJÍA-TERÁN, C. Brucellosis in México: Current status and trends. Veterinary Microbiology. 90: 2002. 19-30p.

LAURIA, I.E.N. Identificação de Brucella spp. Por técnicas moleculares Em carcaças de bovinos com lesão sugestiva de brucelose. 2012. 63f. Dissertação apresentada à Universidade Federal de Mato Grosso do Sul, como requisito à obtenção do título de Mestre em Ciência Animal. Área de concentração: Saúde Animal.- Universidade Federal do Mato Grosso do Sul, Mato Grosso do Sul, 2012.

MAQUART, M.; LE FLĖCHE, P.; FOSTER, G.; TRYLAND, M.; RAMISSE, F.; DJONNE, B.; AL DAHOUK, S.; JACQUES, I.; NEUBAUER, H.; WALRAVENS, K.; GODFROID, J.; CLOECKAERT, A.; VERGNAUD, G. MLVA-16 typing of 295 marine mammal Brucella isolates from different animal and geographic origins indetifies 7 major groups within Brucella ceti and Brucella pinnipedialis. BMC Microbiology, Nouzilly, p.1-11, 20 jul. 2009. Disponível em: <http://www.biomedcentral.com/1471-2180/9/145>. Acessado em 16 julho 2013.

MATHIAS, L.A.; CHAVES, L.F.; CHEN, A.A.; GIRIO, R.J.S.; VALÉRIO, W.N. Evolução de títulos sorológicos, nas provas de soroaglutinação em placa, antígeno acidificado tamponado e fixação de complemento, em bezerras Nelore vacinadas aos 18 meses de idade com Brucella abortus amostra B 19. Pesquisa Veterinária Brasileira, v. 21, n. 4, p.139-142, 2001.

MEGID, J.; RIBEIRO, M.G.; MARCOS, G.J.; CROCCI, A.J. Avaliação das provas de soroaglutinação rápida, soroaglutinação lenta, antígeno acidificado e 2-mercaptoetanol no diagnóstico da brucelose bovina. Brazilian Journal Veterinary. Research Animal Science, v. 37 , n. 5 , p.110-114, 2000.

MOLNÁR, E.; MOLNÁR, L.; DIAS, H.L.T.; SOUZA, J.S.; VALE, W.G. Ocorrência de brucelose bovina no Estado do Pará confirmada por testes sorológicos. Revista Brasileira Medicina Veterinária. v. 22. n. 3, p.117-121. 2000.

MONTEIRO, L.A.R.C. Prevalência e fatores de risco associados à brucelose bovina em rebanhos de Mato Grosso do Sul. 2004. 44f. Dissertação (Mestrado Ciência Animal- Área de concentração animal- Saúde Animal) - Universidade Federal do Mato Grosso do Sul, Mato Grosso do Sul, 2004.

MOTA, M.S. Ocorrência de brucelose bovina no estado do Espírito Santo no período de 2004 a 2007. 2008. Trabalho monográfico de conclusão do curso de Pós-graduação "Latu sensu" (Especialista em Defesa e Vigilância Sanitária Animal). Universidade de Castelo Branco. Vitória-ES, 2008.

NICOLETTI, P. The epidemiology of bovine brucellosis. Advances in Veterinary Science \& Comparative Medicinev. 24, p. 69-98, 1980.

NICOLETTI, P. Uma pequena história da brucelose. Microbiologia Veterinária. v. 90, p. 5-9, 2002.

NIELSEN, K. Diagnosis of brucellosis by serology. Veterinary Microbiology, v. 90, p.447459, 2002.

OLASCOAGA, C.R.C. Diagnóstico serologico de la brucelosis. Zoonosis, v. 18, p.107-141, 1976. 
OMS. Comité Misto FAO/OMS de especialistas em brucelose. Organização Mundial da Saúde, Genebra.Sér. Inf. Téc. p. 740-149. 1986.

PACHECO, G.; MELLO, M.T. Brucelose Humana no Brasil: para o Estudo Nacional da Casuística. Contribuição Mem. Inst. Oswaldo Cruz, Rio de Janeiro, 1950. Disponível em:

$<w w w . s c i e l o . b r / s c i e l o . p h p ?$ script $=$ sci_arttext\&pid $=$ S00740246195000000015\&Ing $=$ en\&nrm $=$ is o>. Acessado em 16 de julho de 2013.

PAULIN, L.M.; NETO, J.S.F. O combate à brucelose bovina. Situação brasileira. $1^{\text {a }}$ ed., São Paulo, Brasil, Editora Funep, 2003, p.154.

PAHO - PAN AMERICAN HEALTH ORGANIZATION. Zoonoses and

Communicable diseases common to man and animals. 3. ed. Washington: World Health Organization, 2001. 378 p.

POESTER, F.P.; GONÇALVES, V.S.P.; LAGE, A.P. Brucellosis in Brazil.

Veterinary Microbiology, v.90, n.1-4, p. 55-62, 2002.

POESTER, F.P.; SAMARTINO, L.E.; LAGE, A.P. Diagnóstico da brucelose bovina. Caderno Técnico Veterinária Zootecnia, n.47, p.13-29, 2005.

POESTER, F.P. Manual de Zoonoses: Brucelose. Curitiba: CRMV(PR,SC, RS), 2009.

SANTOS, R.L.; MARTINS, T.M.; BORGES, A.M.; PAIXÃO, T.A. Economic losses due to bovine brucellosis in Brazil. Pesquisa Veterinária Brasileira. v. 33, n.6, p.759-764, 2013.

SIKUSAWA S. Prevalência e caracterização epidemiológica da Brucelose Bovina do Estado de Santa Catarina. 2004. 107 f. Dissertação (Mestrado em Medicina Veterinária). Faculdade de Medicina Veterinária e Zootecnia, Universidade de São Paulo, São Paulo, 2004.

SIKUSAWA, S.; AMAKU, M.; DIAS, R.A.; FERREIRA NETO, J.S.; MARTINS, C.; GONÇALVES, V.S.P.; FIGUEIREDO, V.C.F.; LÔBO, J.R.; FERREIRA, F. Situação epidemiológica da brucelose bovina no Estado de Santa Catarina. Arquivo Brasileiro de Medicina Veterinária e Zootecnia. supl. 1, v.61, p.103-108, 2009.

SAMARTINO, L.E.; ENRIGHT, F.M. Patogenesis of abortion of bovine brucelosis. Comparative Immunology Microbiology Infectious Disease, v.16, p. 95-101, 1993.

TOLEDO, M.P. Centro Universitário Anhanguera, Brucelose bovina: Vacinação de bezerras entre 3 e 8 meses de idade no município de Santa Cruz da Conceição. p. 2, 2005.

TOLÊDO, K.A. Brucelose bovina. 2006. Trabalho monográfico de curso de pós-graduação "Lato sensu" em Produção e Reprodução de Bovinos. Universidade de Castelo Brando. Brasília- DF. 